\title{
Tratamiento quirúrgico de la periodontitis en un paciente con obesidad. Reporte de caso
}

\section{Surgical treatment of periodontitis in an obese patient. A case report}

\section{Resumen}

Se presenta el caso clínico de una paciente mujer de 39 años con diagnóstico de obesidad clase 1 y periodontitis generalizada que acudió al posgrado de Periodoncia para reducir el "sangrado de sus encías". Al examen clínico se evidenció inflamación generalizada del margen gingival y papilas interdentales, profundidad de sondaje promedio de $3,8 \mathrm{~mm}$, pérdida de inserción clínica promedio de $3,9 \mathrm{~mm}$ y lesiones de furcación clase 1 . Se planificó un tratamiento periodontal mecánico evidenciándose mejora en los parámetros clínicos al cabo de seis meses, sin embargo, persistieron las bolsas periodontales por lo que se indicó terapia periodontal quirúrgica resectiva a través de colgajos de Widman modificado. Con un seguimiento de dos años se evidenció mantenimiento de la salud periodontal con una profundidad al sondaje media de $2 \mathrm{~mm}$ sangrado al sondaje de $6 \%$ e índice de higiene oral de $11 \%$, así también presentar zonas con profundidad de sondaje $>3 \mathrm{~mm}$ pero sin sangrado al sondaje en dichas zonas. Se concluye que luego de una terapia periodontal quirúrgica resectiva, los parámetros periodontales de inflamación gingival, profundidad al sondajes y sangrado al sondaje fueron reducidos y controlados en una paciente diagnosticada con obesidad clase 1 y periodontitis generalizada.

Palabras clave: Periodontitis; Obesidad; Raspado dental; Pérdida de hueso alveolar (fuente: DeCS BIREME).

\begin{abstract}
Clinical case: 39 -year-old female patient with class 1 obesity and generalized periodontitis diagnosis, attended the Postgraduate Periodontology program to reduce "bleeding from her gums". The clinical examination revealed generalized inflammation of the gingival margin and interdental papillae, an average probing depth of $3.8 \mathrm{~mm}$, an average clinical attachment loss of $3.9 \mathrm{~mm}$ and class 1 furcation lesions. A mechanical periodontal treatment was planned, showing improvement in the clinical parameters after six months, however, the periodontal pockets persisted, for which resective surgical periodontal therapy was indicated through modified Widman flaps. With a two-year follow-up, periodontal health was maintained with a mean probing depth of $2 \mathrm{~mm}$, bleeding on probing of $6 \%$ and oral hygiene index of $11 \%$, as well as presenting areas with probing depth> $3 \mathrm{~mm}$ but without bleeding on probing in these areas. It is concluded that after resective surgical periodontal therapy, the periodontal parameters of gingival inflammation, depth to probing and bleeding on probing were reduced and controlled in a patient diagnosed with class 1 obesity and generalized periodontitis.
\end{abstract}

Keywords: Periodontitis; Obesity; Dental scaling; Alveolar bone loss (source: MeSH NLM).
ISSN-L 1560-9111; eISSN: 1609-8617

\section{Caso Clínico}

Jesús Abel Ramírez Guillen 1,a

${ }^{1}$ Universidad Nacional Mayor de San Marcos, Especialidad de Periodoncia, Lima, Perú.

a Cirujano Dentista.

\section{Correspondencia:}

Jesús Abel Ramírez Guillen: ramirezjesus.odo@gmail.com Mz.G lote 53 San Remo 2. San Martín de Porres. Lima. Perú

ORCID: 0000-0002-1233-1021

\section{Editor:}

Yuri Castro Rodríguez

Universidad Nacional Mayor de San Marcos, Perú.

Conflicto de intereses: el autor declara no tener conflicto de intereses.

Fuentes de financiamiento: financiado por el autor.

Recibido: 22/09/20

Aceptado: $01 / 11 / 20$

Publicado: 15/02/21 


\section{Introducción}

La periodontitis es una enfermedad multifactorial de etiología bacteriana en la cual se modifica la respuesta inflamatoria desarrollada por el huésped frente a los microorganismos de la biopelícula dental, sus características clínicas incluyen la pérdida de soporte periodontal, pérdida ósea alveolar, presencia de bolsas periodontales y sangrado al sondaje. Ocasionando un proceso inflamatorio que influye en enfermedades cardiovasculares y metabólicas, entre otras ${ }^{1}$. En taller mundial del 2017 se establecieron al tabaquismo y el control metabólico de la diabetes como factores de riesgo, capaces de afectar la tasa de progresión de la periodontitis ${ }^{2}$. También se considera a la obesidad como indicador de riesgo emergente capaz de contribuir en un futuro a la evaluación y diagnóstico de la periodontitis ${ }^{3}$.

La obesidad es una de las enfermedades crónicas no transmisibles de mayor prevalencia a nivel global (52\% de la población mundial) y sus complicaciones (diabetes tipo 2, enfermedad cardiovascular, hígado graso no alcohólico, etc.) generan un incremento de los costos mundiales en la salud y atención médica ${ }^{4}$. Se caracteriza por un estado de inflamación sub clínica o inflamación crónica de bajo grado en el que se secretan mayores cantidades de citoquinas proinflamatorias (IL- $1 \beta$, IL-6 y FNT $\alpha$ ) incremento que se da en proporción a la cantidad de grasa en el tejido adiposo, modificando la respuesta del anfitrión a los antígenos derivados de la biopelícula dental ${ }^{5}$. Estos cambios hacen que las personas obesas tengan 2-3 veces más probabilidades de sufrir periodontitis independientemente de los factores de riesgo ${ }^{6}$. Además, la hiperlipidemia que frecuentemente acompańa a la obesidad, juega un papel importante en la relación bidireccional entre la diabetes y periodontitis ${ }^{7}$.

Los avances que se dan en el tratamiento de la periodontitis están orientados al control de la infección, así como modulación de la respuesta inmune del hospedero y el control de factores de riesgo. Estos incluyen instrucciones de higiene oral y terapéutica no quirúrgica, a veces complementado con procedimientos quirúrgicos. La terapia periodontal no quirúrgica en pacientes obsesos evidencia mejoras en los parámetros clínicos, sin embargo, existe discrepancia sobre la respuesta periodontal de estos pacientes ${ }^{8}$. Por su parte, la terapia quirúrgica reduce la pérdida de inserción y la profundidad de las bolsas periodontales, sin embargo, esto se encuentra más documentado en lesiones periodontales profundas ${ }^{9}$.

Debido a la creciente prevalencia de la obesidad y el creciente número de cirugías para reducir el peso, es necesario evaluar el estado bucal de estos individuos y cómo las distintas terapias periodontales pueden disminuir los parámetros de inflamación. De esta forma, en el presente reporte de caso se expone los resultados clínicos del tratamiento quirúrgico periodontal de un paciente obeso con diagnóstico de periodontitis generalizada a quien se le realizó un seguimiento de dos años.

\section{Reporte del caso}

Se presenta el caso clínico de una paciente de 39 años de origen mestizo que acudió a la clínica de Periodoncia del Posgrado de la Facultad de Odontología de la Universidad Nacional Mayor de San Marcos (Lima. Perú) en el ańo 2018. La paciente fue una comerciante que acudió aquejando sangrado de encías de larga data aproximadamente 10 años, el mismo que persistía a pesar de haberse realizado profilaxis dental en consultorios particulares, sin embargo, hace aproximadamente 1 ańo el sangrado se incrementó en diversas zonas, asociado a mal aliento. A la consulta manifestó padecer de obesidad con una masa corporal $72 \mathrm{Kg}$, medir 1,55 $\mathrm{m}$, también refirió cefaleas esporádicas motivo por el que acudió a consulta médica siendo diagnosticada con obesidad clase 1 (diagnosticada por índice de masa corporal=peso $/$ altura $^{2}=30$ ), dislipidemia e hipercolesterolemia, presentar un estilo de vida sedentario; indicó que se cepillaba dos veces al día, solo usaba cepillo y pasta dental

Al examen extraoral se evidenció un patrón braquifacial, hipotonicidad muscular, altura de sonrisa baja, sin otros datos contribuyentes. Al examen intraoral se observó una arcada superior trapezoidal e inferior oval, encía adherida con ausencia de puntillado, encía marginal hipertrófica, eritematosa, con sangrado al sondaje en múltiples zonas, recesiones gingivales clase I múltiples (según la clasificación de Miller), pérdidas papilares en zona anterior, mordida bis a bis, movilidad grado II (según la clasificación de Miller) de forma localizada y depósitos de biopelícula calcificada en zonas molares. Al examen clínico periodontal por sextantes se evidenció: múltiples zonas con profundidad de sondaje $>4 \mathrm{~mm}$ (profundidad máxima de $9 \mathrm{~mm}$ ), sangrado al sondaje en el $93 \%$ de las zonas evaluadas, profundidad de sondaje media de $3,8 \mathrm{~mm}$, un índice de higiene oral de $60 \%$ y lesiones de furcación grado I (Tabla).

Se realizó un examen clínico periodontal completo, que incluyó radiografías periapicales seriadas con técnica paralela donde se evidencia destrucción ósea horizontal así como pruebas hematológicas (hemograma completo sin cambios contribuyentes y perfil lipídico con valores elevados de triglicéridos y colesterol). Se diagnosticó periodontitis estadio 3, tomando como criterios de severidad que la paciente presentó una pérdida de inserción clínica $\geq 5 \mathrm{~mm}$ así como pérdida ósea radiográfica que se extendió al tercio medio y como criterio de complejidad una profundidad de sondaje $\geq 6 \mathrm{~mm}$; lo que respecta al riesgo de progresión de periodontitis se clasificó a la paciente con grado B, ya que al no contar con periodontogramas o radiografías anteriores (evidencia directa del riesgo de progresión) se recurrió a la evidencia indirecta de progresión determinada por el porcentaje de pérdida ósea dividida entre la edad del paciente, obteniendo un valor entre 0,25 a $1(0.89)$, no estuvieron presentes los actuales factores de riesgo considerados capaces de modificar el grado de progresión (tabaco y diabetes no controlada) (Figura 1). 
Se planificó una terapia periodontal de fase I que incluyó: educación y motivación a la paciente, control de biopelícula dental de inicio de 60\%, instrucción de higiene oral (técnica de cepillado Bass modificado y uso de hilo dental), raspaje de cálculo supragingival. En las primera reevaluación al cabo de tres meses se verificó que el índice de biopelícula bajase a un valor menor a $20 \%$, por lo que se complementó con raspaje y alisado radicular subgingival por cuadrantes, eliminación de factores retenedores de biopelícula (pulido de resina desbordante en una molar inferior, y exodoncia de terceras molares superiores por ser de difícil acceso para la higiene oral, se esperó un tiempo de seis meses para realizar una segunda reevaluación (Figura 2)

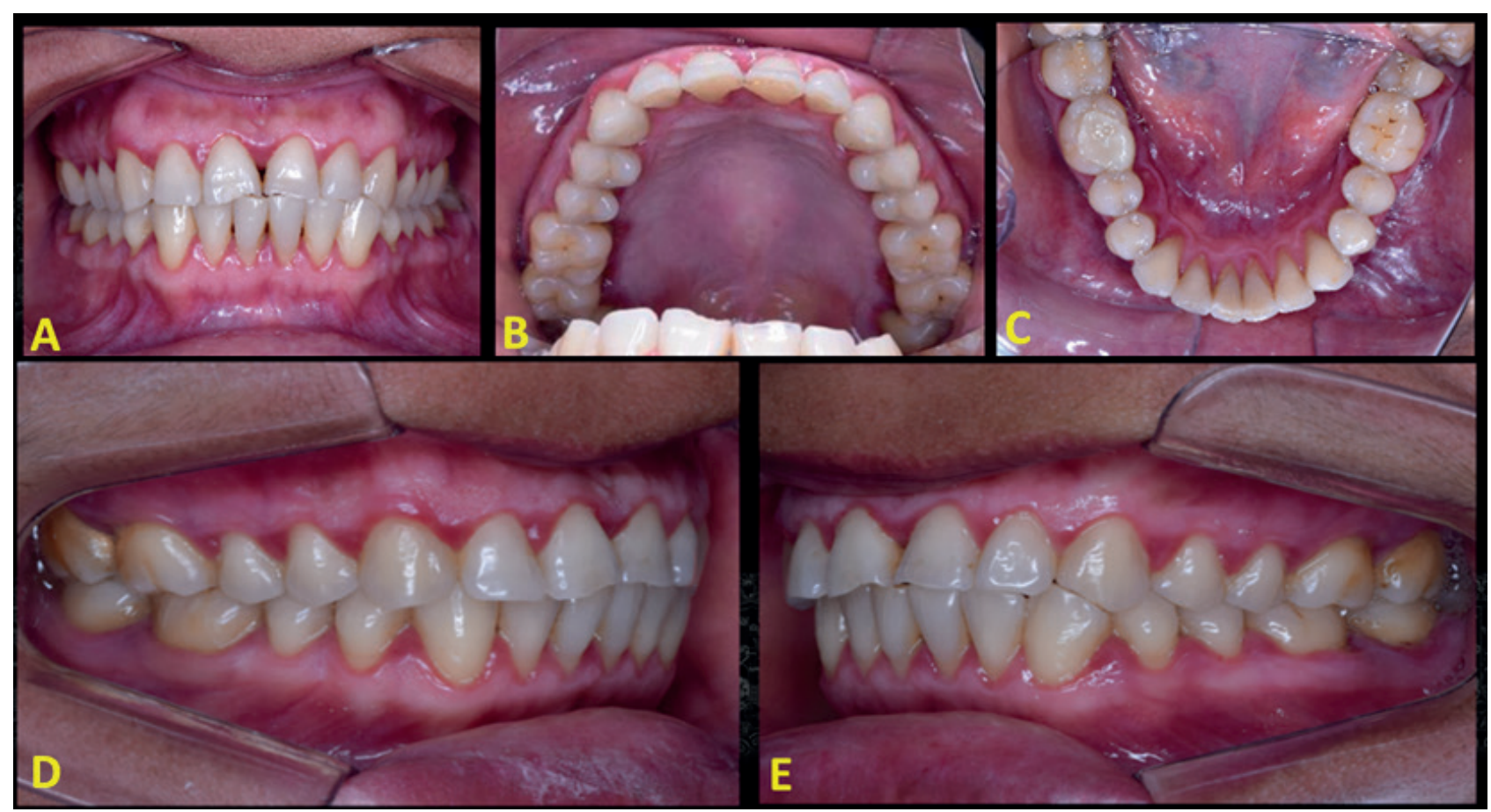

Figura 1. A. Vista frontal con pérdida interdental en el sector anterior. B. Vista oclusal inferior. C. Vista oclusal inferior con restauración desbordante. D. Vista lateral izquierda que evidencia eritema generalizado. E. Vista lateral derecha que evidencia encía lisa

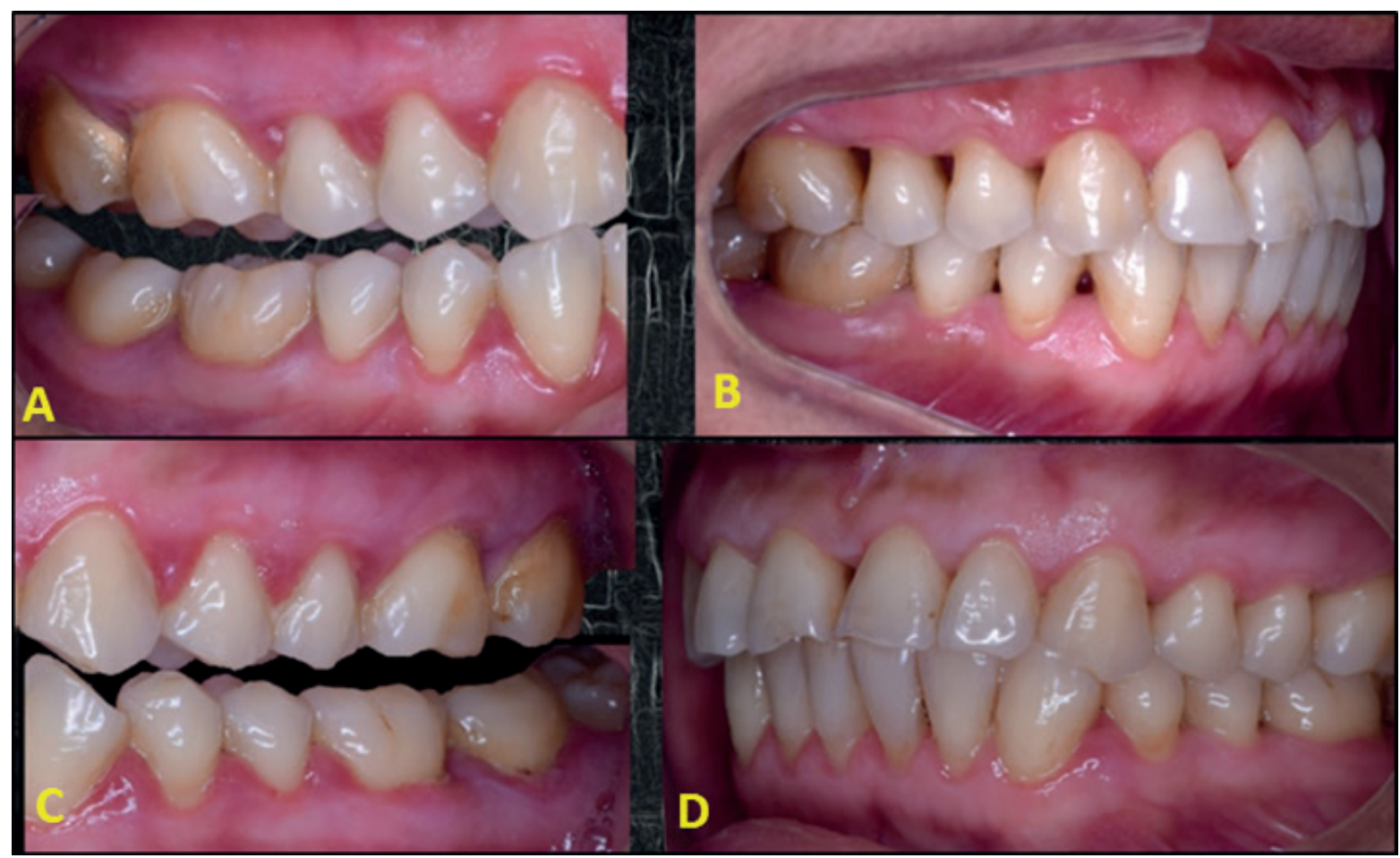

Figura 2. A. Periodonto izquierdo inicial con signos de inflamación. B. Periodonto luego de seis meses de reevaluación de la terapia no quirúrgica. C. Periodonto inicial derecho con acúmulo de biopelícula. D. Vista posoperatoria de la zona derecha sin signos de inflamación 
En la reevaluación, a pesar de haber presentado bolsas con profundidad de sondaje $\geq 6 \mathrm{~mm}$, el tratamiento periodontal no quirúrgico evidenció una disminución del eritema y edema inicial, el sangrado al sondaje disminuyó de $93 \%$ a 4\%, disminución significativa de sitios con profundidad al sondaje 4-5 $\mathrm{mm}$ y $\geq 6 \mathrm{~mm}$ así como una disminución de la profundidad al sondaje media de 3,8 $\mathrm{mm}$ a 2,2 $\mathrm{mm}$, sin embargo, se observó la persistencia de bolsas periodontales localizadas con sangrado al sondaje y un índice de higiene oral de 9\% por lo que planificó una terapia quirúrgica que incluyó cirugía resectiva en molares superiores con la técnica de colgajo de Widman modificado: se procedió a infiltrar con anestésico local, lidocaína $2 \%$ con epinefrina 1:80 000 (Scandicaine ${ }^{\circ}$ ), se realizó incisión a bisel interno de 1 a 1,5 mm del margen gingival, decolado a espesor total deflexión parcial, incisión sulcular e interdentaria, retiro del collarín gingival, alisado de la superficie del cemento radicular, osteoplastía de la pared del defecto óseo y sutura en ocho con ácido poliglicólico 4/0. Se indicó antibioticoterapia con azitromicina $500 \mathrm{mg}$ (Azitral-500 ${ }^{\circ}$ ) cada $24 \mathrm{~h}$ por 3 días y medicación analgésica con ibuprofeno $400 \mathrm{mg}$ (Doloflam extra forte ${ }^{\circledR}$ ) (Figura 3).

Con el objetivo de prevenir la progresión de la periodontitis, la paciente ingresó a terapia periodontal de soporte en la cual se realizó la eliminación de la biopelícula supragingival, cálculo y biopelícula subgingival, además de la reinstrucción de higiene oral, según Laleman y cols ${ }^{10}$ la terapia de soporte se debe dar a intervalos personalizados según la necesidad del paciente, se estableció un intervalo de tres meses; se realizó el seguimiento del paciente por dos años, se evidenció mantenimiento de la salud periodontal con una profundidad al sondaje media de $2 \mathrm{~mm}$ sangrado al sondaje de $6 \%$ e índice de higiene oral $11 \%$, así también presentar zonas con profundidad de sondaje $>3 \mathrm{~mm}$ pero sin sangrado al sondaje en dichas zonas (Figura 4 y Tabla).

\section{Discusión}

La periodontitis es una enfermedad que desencadena una respuesta inflamatoria del hospedador produciendo destrucción de tejidos de soporte del diente con las secuelas tanto estéticas como estructurales ocasionando según la severidad, niveles de inflamación sistémica que influyen en enfermedades cardiovasculares y metabólicas ${ }^{1}$. En el presente reporte de caso se presentó el tratamiento periodontal mecánico y quirúrgico de una periodontitis generalizada y un seguimiento de dos años. Nuestro caso presentó a una paciente con diagnóstico de obesidad clase 1, hipercolesterolemia e hiperlipidemia; características que hicieron de difícil pronóstico los resultados del tratamiento, esto debido a que la evidencia científica indica resultados controversiales ${ }^{11,12}$.

La literatura científica relaciona la presencia de grasa abdominal (tejido adiposo visceral que debido a su metabolismo más activo) con una mayor secreción de citoquinas proinflamatorias que el tejido adiposo subcutáneo ${ }^{13}$, esto implica que los procesos inflamatorios crónicos como la periodontitis son comunes en estos pacientes y las respuestas inmunitarias son más lentas e impredecibles por lo que los tratamientos periodontales (sean mecánicos o quirúrgicos) deben ser constantes y duraderos.

Los procedimientos descritos tanto la terapia periodontal no quirúrgica como la quirúrgica, tienen una amplia literatura médica que sustenta su uso ${ }^{14}$. Sin embargo, todavía existe controversias en los resultados del tratamiento periodontal no quirúrgico en pacientes obesos, existen revisiones sistemáticas que demuestran su efectividad en los parámetros periodontales e incluso en algunos estudios reduciendo los niveles de marcadores proinflamatorios sistémicos ${ }^{15,16}$, sin embargo, otros estudios han encontrado resultados pobres del tratamiento periodontal no quirúrgico en pacientes obesos ${ }^{17}$. Nuestro caso evidenció una reducción de los parámetros

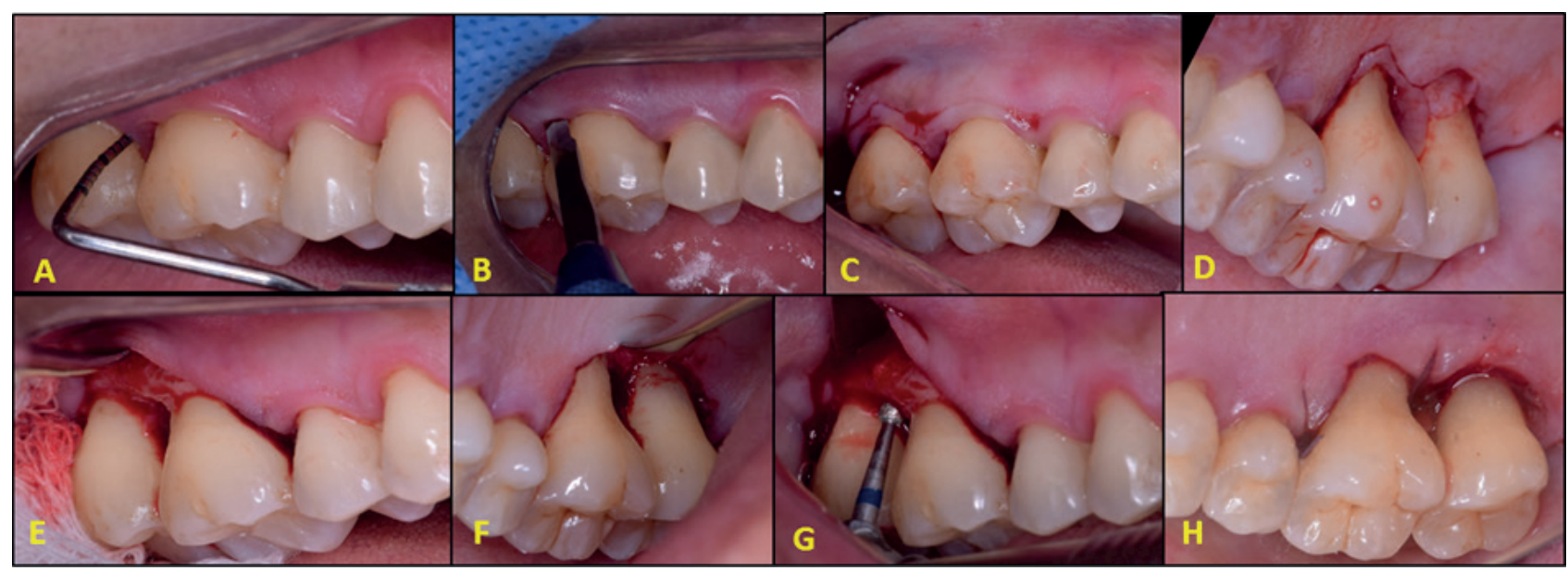

Figura 3. A. Profundidad de sondaje persistente mayor a $4 \mathrm{~mm}$. B. Incisión a bisel interno vestibular. C. Incisión a bisel interno palatino. D. Retiro del tejido gingival. E. Decolado a espesor total por vestibular. F. Decolado a espesor total por palatino. G. Osteotomía con medios rotatorios. H. Sutura con ácido poliglicólico 


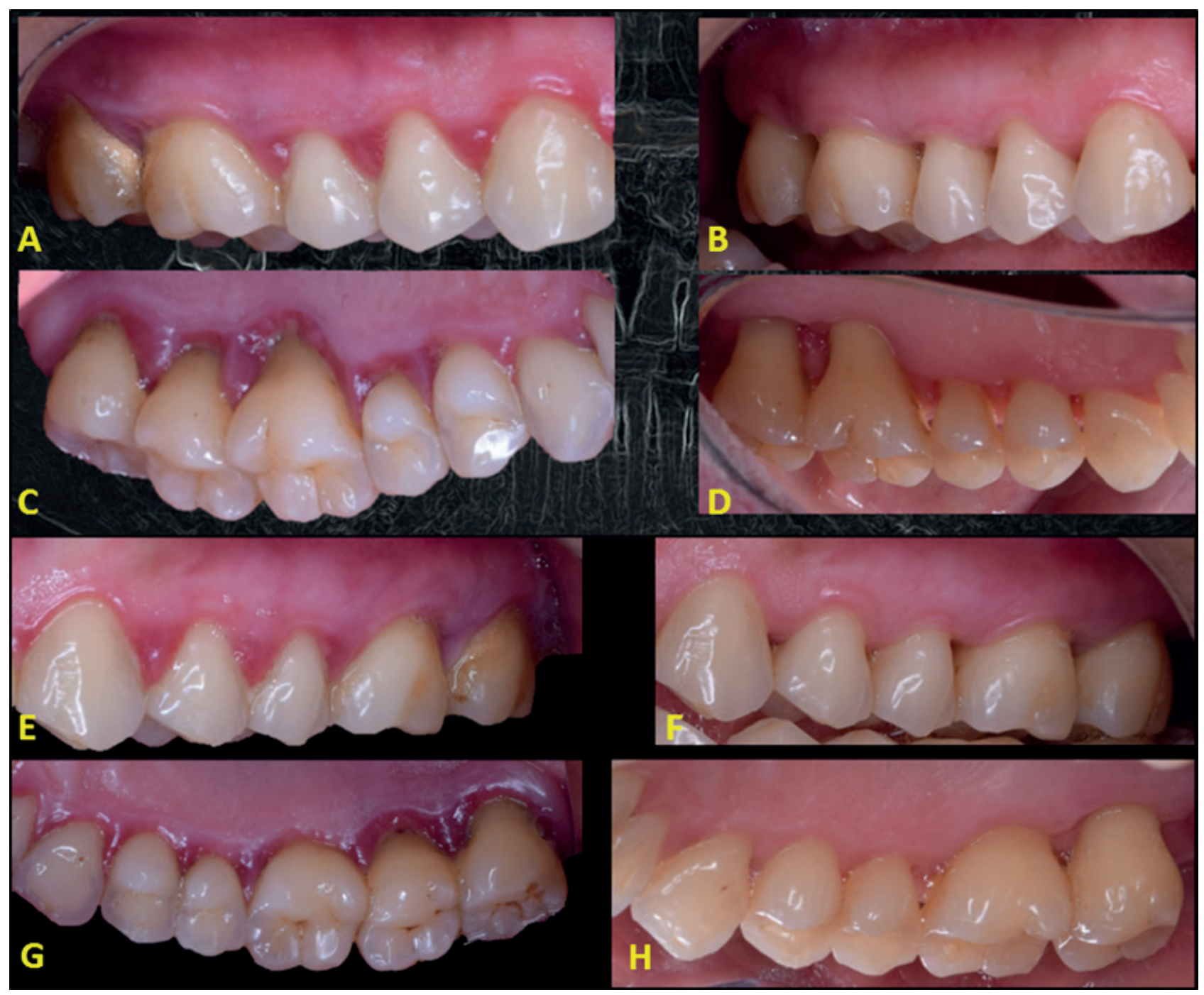

Figura 4. A y C. Vista vestibular/palatina superior derecha inicial. B y D. Periodonto superior derecho por vestibular y palatino luego de dos años de seguimiento. E y G. Vista vestibular/palatina superior izquierda inicial. F y H. Periodonto superior izquierdo por vestibular y palatino luego de dos años de seguimiento

Tabla. Cambios de los parámetros periodontales antes, durante y después del tratamiento quirúrgico

\begin{tabular}{lccc}
\hline Parámetros & Inicial & Seis meses & Dos años de seguimiento \\
\hline PS media (mm) & 3,8 & 2,2 & 2 \\
NAC media (mm) & 3,9 & 2,9 & 2,8 \\
Sitios de PS 1-3mm (\%) & 45,83 & 86,6 & 95 \\
Sitios de PS 4-5mm (\%) & 32,29 & 11,1 & 5 \\
Sitios de PS $\geq 6 \mathrm{~mm}(\%)$ & 21,87 & 2,2 & 0 \\
SS (\%) & 93 & 4 & 6 \\
IHO (\%) & 60 & 9 & 11 \\
\hline
\end{tabular}

PS: profundidad al sondaje. NIC: nivel de inserción clínica. SS: sangrado al sondaje. IHO: índice de higiene oral

inflamatorios luego del tratamiento no quirúrgico, principalmente en la reducción de la inflamación gingival.

Los efectos a nivel sistémico que produce el tratamiento periodontal en pacientes obesos, han evidenciado una disminución de niveles en sangre de marcadores proinflamatorios como FNT $\alpha$, IL-6, y con ello contribuye a disminuir la resistencia a la insulina, mejorando de esa forma el estado sistémico de los pacientes obesos ${ }^{18}$. El presente caso no hace posible evidenciar estas mejoras a nivel de marcadores proinflamatorios sistémicos, al no haberse realizado pruebas de laboratorio para evidenciar estos cambios, sin embargo, se observa mejoras clínicas significativas a nivel de la inflamación de los tejidos gingivales. 
La profundidad inicial de sondaje, anatomía radicular, el diseño de los instrumentos y la habilidad y experiencia del operador influyen en la eliminación completa del cálculo, bacterias y alisamiento de la superficie radicular ${ }^{19-21}$. En el presente caso se evidenció una mejoría clínica evidente de los parámetros periodontales post terapia no quirúrgica, al encontrar disminución de la profundidad al sondaje y sangrado al sondaje resultados que apoyan la posición de una evolución favorable de la terapia periodontal no quirúrgica en pacientes obesos, reduciendo la necesidad de realizar tratamientos quirúrgicos complejos complementarios en la fase II.

La reevaluación de los resultados del tratamiento periodontal no quirúrgico después del tratamiento inicial es esencial para seleccionar adecuadamente la terapia adicional y establecer el mejor pronóstico posible a largo plazo, sin embargo, las bolsas "residuales" ponen en peligro la supervivencia del diente y son determinantes en la progresión de la enfermedad. Una revisión sistemática indicó una profundidad de sondaje $\geq 6 \mathrm{~mm}$ como umbral para el tratamiento quirúrgico de las bolsas periodontales ${ }^{22}$. Además, otros autores coinciden en que las intervenciones quirúrgicas de las lesiones profundas permiten ganar más en términos de reducción de profundidad de sondaje en comparación con un enfoque no quirúrgico ${ }^{23}$. Otras indicaciones del tratamiento quirúrgico son acceso a la superficie de la raíz para el desbridamiento a colgajo abierto, regeneración periodontal caracterizada por la formación de nuevas fibras periodontales del cemento al hueso alveolar y la curación de defectos óseos rellenando con injertos óseos ${ }^{24}$. Por lo cual en el presente caso se optó por un tratamiento quirúrgico localizado de técnica resectiva por la condición económica del paciente; esta opción evidenció resultados favorables con parámetros clínicos estables con un seguimiento de dos años. Además, resulta importante concluir la necesidad de establecer la terapia periodontal de mantenimiento personalizado según el perfil de riesgo del paciente, considerando los factores de riesgo ya conocidos como tabaquismo, diabetes y así también los factores de riesgo emergentes como la obesidad.

Cabe indicar que la terapia quirúrgica más el desbridamiento subgingival induce cambios favorables en los tejidos periodontales, en términos de reducción de la inflamación gingival, reducción de la profundidad de sondaje y la ganancia del nivel de inserción clínica, sin embargo, aunque el desbridamiento subgingival es un método eficaz, ninguna de las técnicas utilizadas actualmente: instrumentación manual, instrumentación ultrasónica, laser terapia fotodinámica y "air-polishing" resultan totalmente eficaces sino se complementan con una terapia de soporte y un estricto cumplimiento de la higiene oral por parte del paciente.

La remoción de la biopelícula dental y un riguroso control de la higiene oral del paciente minimizan la inflamación gingival y permite reducir la profundidad de sondaje en un paciente obeso con diagnóstico de periodontitis. Con un control de dos ańos de seguimiento luego de una terapia periodontal quirúrgica resectiva, los parámetros periodontales de inflamación gingival, profundidad al sondajes y sangrado al sondaje fueron reducidos y controlados en una paciente diagnosticada con obesidad clase 1 y periodontitis generalizada.

\section{Referencias bibliográficas}

1. Slots J. Periodontitis: facts, fallacies and the future. Periodontol 2000. 2017;75(1):7-23.

2. Tonetti M, Greenwell H, Kornman K. Staging and grading of periodontitis: Framework and proposal of a new classification and case definition. J Clin Periodontol. 2018;45(Suppl 20):149-61.

3. Khan S, Barrington G, Bettiol S, Barnett T, Crocombe L. Is overweight/obesity a risk factor for periodontitis in young adults and adolescents?: a systematic review. Obes Rev. 2018;19(6):852-883.

4. Engin A. The Definition and Prevalence of Obesity and Metabolic Syndrome. Adv Exp Med Biol. 2017;960:1-17.

5. Martinez-Herrera M, Silvestre-Rangil J, Silvestre FJ. Association between obesity and periodontal disease. A systematic review of epidemiological studies and controlled clinical trials. Med Oral Patol Oral Cir Bucal. 2017;22(6):e708-e715.

6. Martens L, De Smet S, Yusof MY, Rajasekharan S. Association between overweight/obesity and periodontal disease in children and adolescents: a systematic review and meta-analysis. Eur Arch Paediatr Dent. 2017 Apr;18(2):69-82.

7. Zhou X, Zhang W, Liu X, Zhang W, Li Y. Interrelationship between diabetes and periodontitis: role of hyperlipidemia. Arch Oral Biol. 2015;60(4):667-74.

8. Papageorgiou SN, Reichert C, Jäger A, Deschner J. Effect of overweight/obesity on response to periodontal treatment: Systematic review and a meta-analysis. J Clin Periodontol. 2015;42(3):247-61.

9. Lang NP, Salvi GE, Sculean A. Nonsurgical therapy for teeth and implants-When and why? Periodontol 2000. 2019;79(1):15-21.

10. Laleman I, Cortellini S, De Winter S, Rodriguez Herrero E, Dekeyser C, Quirynen M, Teughels W. Subgingival debridement: end point, methods and how often?. Periodontol 2000. 2017;75:189-204.

11. Manresa C, Sanz-Miralles EC, Twigg J, Bravo M. Supportive periodontal therapy (SPT) for maintaining the dentition in adults treated for periodontitis. Cochrane Database Syst Rev. 2018;1(1):CD009376.

12. Keller A, Rohde JF, Raymond K, Heitmann BL. Association between periodontal disease and overweight and obesity: a systematic review. J Periodontol. 2015;86(6):766-76.

13. Khader YS, Bawadi HA, Haroun TF, Alomari M, Tayyem RF. The association between periodontal disease and obesity among adults in Jordan. J Clin Periodontol. 2009;36(1):18-24.

14. Adriaens P, Adriaens L. Effects of nonsurgical periodontal treatment on hard and soft tissues. Periodontol 2000. 2005;121-45. 
15. Papageorgiou SN, Reichert C, Jäger A, Deschner J. Effect of overweight/obesity on response to periodontal treatment: Systematic review and a meta-analysis. J Clin Periodontol. 2015;42(3):247-61.

16. Nascimento GG, Leite FR, Correa MB, Peres MA, Demarco FF. Does periodontal treatment have an effect on clinical and immunological parameters of periodontal disease in obese subjects? A systematic review and meta-analysis. Clin Oral Investig. 2015;20(4):639-47.

17. Suvan J, Petrie A, Moles DR, Nibali L, Patel K, Darbar $\mathrm{U}$, et al. Body mass index as a predictive factor of periodontal therapy outcomes. J Dent Res. 2014;93(1):49-54.

18. Altay U, Gürgan C, Ağbaht K. Changes in inflamatory and metabolic parameters after periodontal treatment in patients with and without obesity. J Periodontol. 2013;84(1):13-23.

19. Bertl K, Parllaku A, Pandis N, Buhlin K, Klinge B, Stavropoulos A. The effect of local and systemic statin use as an adjunct to non-surgical and surgical periodontal therapy-A systematic review and meta-analysis. J Dent. 2017;67:18-28.
20. Behdin S, Monje A, Lin G, Edwards B, Othman A, Wang H. Effectiveness of laser application for Periodontal Surgical therapy: systematic review and meta-analysis. J periodontol 2015;12:1352-63.

21. Zhang J, Liu J, Li J, Chen B, Li H, Yan F. The Clinical Efficacy of Subgingival Debridement by Ultrasonic Instrumentation Compared With Subgingival Air Polishing During Periodontal Maintenance: A Systematic Review. J Evid Based Dent Pract. 2019;19(4):101314.

22. Salhi L, Albert A, Seidel L, Lambert F. Respective Effects of Oral Hygiene Instructions and Periodontal Nonsurgical Treatment (Debridement) on Clinical Parameters and Patient-Reported Outcome Measures with Respect to Smoking. J Clin Med. 2020;9(8):2491.

23. Serino G, Rosling B, Ramberg P, Socransky S, Lindhe J. Initial outcome and long-term effect of surgical and non-surgical treatment of advanced periodontal disease. J Clin Periodontol 2001:28:910-6.

24. Lang N. Focus on intrabony defects-conservative therapy. Periodontol 2000. 2000;22:51-58. 
